\title{
Supplementary Information for "Analytical
}

\section{Theory of Thin-Film Schottky Diodes"}

\author{
Joshua Wilson, ${ }^{\dagger}$ Jiawei Zhang, ${ }^{\dagger}$ and Aimin Song*, ${ }^{*}, \ddagger$ \\ $\dagger$ †chool of Electrical and Electronic Engineering, University of Manchester, Manchester, \\ M13 9PL, United Kingdom \\ $\ddagger$ Center of Nanoelectronics and Microelectronics, Shandong University, Jinan 250100 , \\ People's Republic of China \\ E-mail: a.song@manchester.ac.uk
}

\section{Diffusion current}

The diffusion equation states that

$$
J=q\left(n_{e} \mu_{n} \mathcal{E}+D_{n} \frac{d n_{e}}{d z}\right)
$$

where $J$ is the current density in the $z$-direction, $n_{e}$ is the free electron concentration, $\mu_{n}$ is the electron mobility and $D_{n}=\frac{k T}{q} \mu_{n}$ is the Einstein relation. As $\mathcal{E}=\frac{1}{q} \frac{d E_{c}}{d z}$,

$$
J=q D_{n}\left(\frac{n_{e}}{k T} \frac{d E_{c}}{d z}+\frac{d n_{e}}{d z}\right)
$$

Hence, the diffusion equation can be integrated using $\frac{E_{c}(z)}{k T}$ as an integrating factor to give:

$$
\int_{0}^{H} J \exp \left(\frac{E_{c}(z)}{k T}\right) d z=\left.\right|_{0} ^{H} q D_{n}\left[\exp \left(\frac{E_{c}(z)}{k T}\right) n_{e}(z)\right]
$$


Given that the current should be constant along a one-dimensional cross section of the device and that the following boundary conditions apply:

$$
E_{C}(z)= \begin{cases}q \phi_{B} & z=0 \\ q\left(\phi_{n}+V\right) & z=H\end{cases}
$$

and

$$
n_{e}(z)= \begin{cases}N_{C} \exp \left(-\frac{q \phi_{B}}{k T}\right) & z=0 \\ N_{C} \exp \left(-\frac{q \phi_{n}}{k T}\right) & z=H\end{cases}
$$

Hence,

$$
J=\frac{q D_{n} N_{C} \exp \left[\exp \left(\frac{q V}{k T}\right)-1\right]}{\int_{0}^{H} \exp \left(\frac{E_{c}(z)}{k T}\right) d z} .
$$

Substituting in the value for $E_{C}$ (Eq. 5) gives

$$
J=\frac{q D_{n} N_{C} \exp \left(-\frac{q \phi_{B}}{k T}\right)\left[\exp \left(\frac{q V}{k T}\right)-1\right]}{\int_{0}^{H} \exp \left[\frac{q^{2} N_{D}}{2 \epsilon_{0} \epsilon_{s} k T} z^{2}-\left(\frac{q\left(\phi_{b i}-V\right)}{k T H}+\frac{q^{2} N_{D}}{2 \epsilon_{0} \epsilon_{s} k T} H\right) z\right] d z} .
$$

From Eq. 6, the integral in the denominator can be rewritten as:

$$
\text { Int. } \approx \int_{0}^{H} \exp \left[\frac{q \mathcal{E}(z)}{k T} z\right] d z
$$

The electric field is quite constant throughout the semiconductor, as shown by the linearity of the conduction band in Fig. 2. Thus, $\mathcal{E}(z)$ can be replaced with $\mathcal{E}(0)$ without significantly reducing the accuracy of the model, giving

$$
\text { Int. } \approx \int_{0}^{H} \exp \left[\frac{q \mathcal{E}(0)}{k T} z\right] d z \approx\left[\frac{q \mathcal{E}_{M T}}{k T}\right]^{-1}
$$


Thus, the current density can be expressed as

$$
J \approx q \mu_{n} N_{C} \mathcal{E}_{M T} \exp \left(-\frac{q \phi_{B}}{k T}\right)\left[\exp \left(\frac{q V}{k T}\right)-1\right]
$$

which is comparable to the standard diffusion equation, except that $\mathcal{E}_{M}$ is replaced by $\mathcal{E}_{M T}$.

\section{Current through a barrier with multiple inhomogeneities}

Using a Gaussian distribution leads to divergent integrals when calculating the current, so a Gamma distribution with a probability density function of

$$
P(\theta)=\frac{\theta^{\kappa-1}}{\Gamma(\kappa) w^{\kappa}} \exp \left(-\frac{\theta}{w}\right) \quad \theta \geq 0
$$

was selected instead. For ease of calculation the parameters were chosen as $w=2 \xi^{2} / \theta$ and $\kappa=3 / 4$, giving

$$
P(\theta)=\frac{2 c \sqrt{\theta}}{\Gamma\left(\frac{3}{4}\right)\left(2 \xi^{2}\right)^{3 / 4}} \exp \left(-\frac{\theta^{2}}{2 \xi^{2}}\right) \quad \theta \geq 0,
$$

where $\xi$ is a measure of the distribution width. Integrating the current over all possible $\theta$ yields

$$
\begin{aligned}
I= & \int_{0}^{\infty} A A^{*} T^{2} \exp \left\{\frac{-q \phi_{B}^{0}}{k T}\right\}\left[\exp \left\{\frac{q V}{k T}\right\}-1\right] \\
& \times\left[1+\frac{1}{L_{x}}\left[\frac{k T}{q}\right]^{1 / 2}\left[\frac{\pi}{\mathcal{E}_{M T}^{3}}\right]^{1 / 4} \theta^{1 / 2} \exp \left\{\frac{2 q \theta \sqrt{\frac{\mathcal{E}_{M T}}{\pi}}}{k T}\right\} P(\theta)\right] d \theta \\
= & A A^{*} T^{2} \exp \left\{\frac{-q \phi_{B}^{0}}{k T}\right\}\left[\exp \left\{\frac{q V}{k T}\right\}-1\right] \\
& \times\left[1+\frac{2 c}{\Gamma\left(\frac{3}{4}\right) L_{x}\left(2 \xi^{2}\right)^{3 / 4}}\left[\frac{k T}{q}\right]^{1 / 2}\left[\frac{\pi}{\mathcal{E}_{M T}^{3}}\right]^{1 / 4} \int_{0}^{\infty} \theta \exp \left\{\frac{2 q \theta \sqrt{\frac{\mathcal{E}_{M T}}{\pi}}}{k T}-\frac{\theta^{2}}{2 \xi^{2}}\right\} d \theta\right] .
\end{aligned}
$$


Here, $L_{x}$ is the width of the diode and $c$ behaves as a quasi-density parameter for low barrier regions. It has been assumed that the contribution of the higher barrier region is small enough that their area can be assumed to be the full area of the diode. Solving the integral and simplifying gives Eq. 50 . 